6. La Commission apprend avec satisfaction qu'une commission a été créée par la Royal Society de Londres pour assurer la publication des lettres de Newton, publication qu'elle souhaitait dans sa recommandation 8 prise lors du Congrès de Stockholm en I938.

\title{
Commission 6. (TÉLÉGRAMMES)
}

I. La Commission recommande qu'une subvention annuelle de mille deux cents francs-or soit allouée au Bureau des Télégrammes astronomiques.

2. La Commission recommande que le code des télégrammes astronomiques (p. 477 ou 483 des Transactions of the I.A.U.6, I938), soit modifié de la manière suivante:

( $\left.\mathrm{I}^{\circ}\right)$ nom de l'auteur de la découverte;

$\left(2^{\circ}\right)$ nature de l'objet;

$\left(3^{\circ}\right)$ nom de l'observateur;

(4 ${ }^{\circ}$ groupes habituels donnant la position, suivis de remarques, s'il y a lieu;

$\left(5^{\circ}\right)$ noms des transmetteurs.

Le Bureau Central des Télégrammes astronomiques décidera s'il convient de communiquer aussi le nom de l'observatoire, ainsi que les noms de tous les intermédiaires.

\section{Commission 8. (ASTRONOMIE MÉRIDIENNE)}

La Commission tient à exprimer sa satisfaction pour les programmes en cours d'exécution aux Etats-Unis et dans l'Union Soviétique, et qui ont pour but de relier le système des nébuleuses extragalactiques avec celui des étoiles fondamentales. La Commission considère que ce travail sera de la plus grande importance pour l'astronomie de position.

\section{Commission Io. (PHÉNOMÈNES PHOTOSPHÉRIQUES)}

I. La Commission propose d'accorder à l'Observatoire fédéral de Zürich pour la période qui s'étendra jusqu'au prochain congrès une subvention annuelle de S. frs. I500, destinée à la publication des cartes héliographiques de la photosphère, qui sont élaborées avec l'aide d'un grand nombre d'observatoires de divers pays.

2. La Commission propose que l'on change son nom actuel de 'Commission des taches solaires et des nombres caractéristiques' en celui plus approprié aux travaux qu'elle réalise actuellement de 'Commission des phénomènes photosphériques'.

3. La Commission recommande que l'on organise une collaboration internationale, sous la direction de l'Observatoire de Zürich, en vue d'une meilleure investigation du développement des taches solaires, soit par l'utilisation des photographies déjà existantes, soit par l'obtention systématisée de photographies fréquentes des détails des groupes.

\section{Commission II. (PhÉNOMÈnes ChromosPhÉRIQUes et CoURonNe solaIRE)}

I. La Commission recommande que la subvention annuelle de 2700 francs-or accordée par la dernière Assemblée Générale de l'U.A.I. à l'Observatoire de Paris-Meudon pour la publication des cartes synoptiques de la chromosphère solaire, soit renouvelée pour la période qui s'étendra jusqu'au prochain congrès de l'Union.

2. La Commission invite les directeurs des établissements possédant un des spectrohélioscopes standards que G. E. Hale avait fait construire en I930, et qui ne l'utilisent pas actuellement, à le remettre en service pour la recherche des éruptions chromosphériques ou, éventuellement, à le prêter à d'autres observatoires mieux placés pour les utiliser.

3. La Commission s'associe à la recommandation de la Commission mixte du Conseil International des Unions Scientifiques pour l'étude des relations entre les phénomènes solaires et terrestres, relative à l'envoi d'ursigrammes sur l'activité solaire par plusieurs 